\title{
INVITED AND PLENARY LECTURES
}

Gordon Research Conference on Natural Products (Short Talk)

07/08/72

(New Hampton, NH)

Sandoz Pharmaceuticals (Hanover, NJ)

$05 / 30 / 73$

University of Rochester (Rochester, NY)

$10 / 03 / 73$

Manhattan College (Bronx, NY)

$12 / 05 / 73$

Iowa State University (Ames, IA)

$01 / 09 / 74$

University of Pittsburgh (Pittsburgh, PA)

$03 / 20 / 74$

WOSNPC-V (Workshop on Organic Synthesis and Natural Products

$08 / 13 / 74$

Chemistry) sponsored by NSF (Onamia, MN)

Lederle Laboratories of American Cyanamid Corporation (Pearl River, NY)

$11 / 09 / 74$

New York University (New York, NY)

$04 / 04 / 75$

Eli Lilly \& Co. (Indianapolis, IN)

$05 / 01 / 75$

WOSNPC-VI (Gatlinburg, TN)

$08 / 13 / 75$

Rutgers University (New Brunswick, NJ)

$10 / 13 / 75$

Schering Corporation (Bloomfield, NJ)

$11 / 24 / 75$

Brown University (Providence, RI)

$12 / 05 / 75$

Yale University (New Haven, CT)

$02 / 26 / 76$

University of North Carolina (Chapel Hill, NC)

$03 / 22 / 76$

SUNY - Buffalo (Buffalo, NY)

$04 / 29 / 76$

Colorado State University (Fort Collins, CO)

$05 / 03 / 76$

University of Colorado (Boulder, CO)

$05 / 04 / 76$

Eli Lilly \& Co. (Indianapolis, IN)

$05 / 25 / 76$

Wayne State University (Detroit, MI)

$05 / 26 / 76$

North Jersey Section - ACS (Seton Hall University)

$10 / 25 / 76$

The Upjohn Company (Kalamazoo, MI)

$10 / 29 / 76$

Northeastern University (Boston, MA)

$11 / 03 / 76$

Rensselaer Polytechnic Institute (Troy, NY)

$11 / 17 / 76$

Cornell University (Ithaca, NY)

$11 / 22 / 76$

Wesleyan University (Middletown, CT)

$12 / 17 / 76$

Rockefeller University (New York, NY)

Ciba-Giegy Corporation (Summit, NJ) 
Emory University (Atlanta, GA)

Polytechnic Institute of New York (Brooklyn, NY)

Queens College (CUNY) (New York, NY)

Case Western Reserve University (Cleveland, $\mathrm{OH}$ )

University of Connecticut (Storrs, CT)

The Pennsylvania State University (University Park, PA)

$02 / 07 / 78$

University of Michigan (Ann Arbor, MI)

$03 / 02 / 78$

SUNY - Stony Brook (Stony Brook, NY)

$03 / 14 / 78$

USV Pharmaceuticals (Tuckahoe, NY)

$04 / 20 / 78$

Hoffmann-LaRoche (Nutley, NJ)

$04 / 26 / 78$

Merck (West Point, PA)

$05 / 12 / 78$

Sterling-Winthrop Research Institute (Rensselaer, NY)

$05 / 31 / 78$

Gordon Research Conference on Natural Products (New Hampton, NH)

$07 / 26 / 78$

Brooklyn College-CUNY (Brooklyn, NY)

$10 / 25 / 78$

Oregon Graduate Center (Portland, OR)

$03 / 27 / 79$

Washington State University (Pullman, WA)

$03 / 28 / 79$

University of Idaho (Moscow, ID)

$03 / 29 / 79$

WOSNPC-X (Pingree Park, CO)

University of Pennsylvania (Philadelphia, PA)

Temple University (Philadelphia, PA)

ACS Northeastern Section, Medicinal Chemistry Group (Boston College,

Chestnut Hill, MA)

Illinois Institute of Technology (Chicago, IL)

$03 / 12 / 80$

University of Virginia (Charlottesville, VA)

04/08/80

Ciba-Geigy Corporation (Ardsley, NY)

$05 / 05 / 80$

New York Academy of Sciences (New York, NY)

05/06/80

21st Annual SUNY-Buffalo Medicinal Chemistry Symposium (Buffalo, NY)

$05 / 19 / 80$

Ohio State University (Columbus, $\mathrm{OH}$ )

Dynapol Corp. (Palo Alto, CA)

University of California (Berkeley, CA)

Gordon Research Conference on Heterocyclic Chemistry (New Hampton, NH) 
Rochester Section ACS, Synthesis Symposium (Rochester, NY)

$10 / 29 / 80$

ICI, Americas (Wilmington, DE)

$11 / 10 / 80$

University of Maryland (College Park, MD)

$11 / 11 / 80$

University of Wisconsin (Madison, WI)

$02 / 04 / 81$

Searle (Chicago, IL)

$02 / 05 / 81$

DuPont Central Research (Wilmington, DE)

$03 / 05 / 81$

University of Delaware (Newark, DE)

$03 / 06 / 81$

Burroughs Wellcome Company (Research Triangle, NC)

04/08/81

Smith Kline \& French (Philadelphia, PA)

$04 / 13 / 81$

McNeil Pharmaceutical Corporation (Spring House, PA)

$05 / 21 / 81$

WOSNPC-XII (Pingree Park, CO)

$07 / 08 / 81$

University of South Carolina (Columbia, SC)

$10 / 16 / 81$

SUNY-Fredonia (Fredonia, NY)

$10 / 22 / 81$

Merck (Rahway, NJ)

$10 / 28 / 81$

American Cyanamid Company (Princeton, NJ)

$12 / 09 / 81$

Guenther Award Symposium-ACS (Las Vegas, NV)

$03 / 31 / 82$

University of Zurich (Zurich, Switzerland)

$05 / 04 / 82$

Philadelphia Organic Chemists Club (Philadelphia, PA)

$05 / 27 / 82$

ACS Metrochem Synthesis Symposium (New Brunswick, NJ)

$06 / 07 / 82$

Ortho Pharmaceutical Corporation (Raritan, NJ)

$06 / 08 / 82$

Gordon Research Conference on Organic Reactions and Processes

$07 / 19 / 82$

(New Hampton, NH)

Cornell University (Ithaca, NY)

$09 / 20 / 82$

West Virginia University (Morgantown, WV)

$10 / 27 / 82$

Georgetown University (Washington, DC)

Penn State University Medical Center (Hershey, PA)

Squibb (Princeton, NJ)

Stevens Institute of Technology (Hoboken, NJ)

Sandoz (E. Hanover, NJ)

Berlex Laboratories (Cedar Knolls, NJ)

Korea Advanced Institute of Science \& Technology (Seoul, Korea)

Takeda Chemical Industries (Osaka, Japan)

9th International Congress of Heterocyclic Chemistry (Tokyo, Japan) 
Wayne State University (Detroit, MI)

$11 / 01 / 83$

Warner-Lambert Pharmaceutical Co. (Ann Arbor, MI)

$11 / 02 / 83$

The Upjohn Company (Kalamazoo, MI)

$11 / 03 / 83$

University of Cambridge (Cambridge, England)

$02 / 13 / 84$

University of East Anglia (Norwich, England)

$02 / 16 / 84$

Imperial College (London, England)

$02 / 21 / 84$

Wellcome Research Laboratories (Beckenham, England)

$02 / 29 / 84$

Pfizer (Sandwich, England)

03/08/84

Shell (Sittingbourne, England)

03/09/84

Royal Dutch Chemical Society Symposium (Wageningen, Netherlands)

03/16/84

Nijmegen University (Nijmegen, Netherlands)

03/19/84

University of Amsterdam (Amsterdam, Netherlands)

03/20/84

Twente University (Enschede, Netherlands)

$03 / 21 / 84$

University of Groningen (Groningen, Netherlands)

$03 / 23 / 84$

University of Louvain (Louvain, Belgium)

$04 / 13 / 84$

CNRS (Gif-sur-yvette, France)

$04 / 16 / 84$

University of Geneva (Geneva, Switzerland)

04/19/84

University of Konstanz (Konstanz, Germany)

$04 / 24 / 84$

ACS-MARM Symposium (Newark, NJ)

$05 / 21 / 84$

Hoechst-Roussel Corp. (Somerville, NJ)

05/22/84

California Inst. Tech. (Pasadena, CA)

06/06/84

University of California (Irvine, CA)

06/07/84

ACS Medicinal Chemistry Symposium (Tucson, AZ)

$06 / 21 / 84$

American Cyanamid Corp. (Stanford, CT)

$10 / 16 / 84$

Kings College (Wilkes-Barre, PA)

$11 / 15 / 84$

Sterling-Winthrop Research Institute (Rensselaer, NY)

$11 / 16 / 84$

Philadelphia Organic Chemists Club (Philadelphia, PA)

$03 / 28 / 85$

Merck, Sharp and Dohme (West Point, PA)

$03 / 29 / 85$

North Carolina State University (Raleigh, NC)

$04 / 01 / 85$

Hunter College - CUNY (New York, NY)

$04 / 19 / 85$

Pfizer (Groton, CT)

$05 / 21 / 85$

American Cyanamid Symposium (Princeton, NJ) 05/23/85

Rohm and Haas Corp. (Spring House, PA) 
University of Notre Dame (South Bend, Indiana)

$09 / 04 / 85$

Millersville University (Millersville, PA)

$09 / 18 / 85$

Lebanon Valley College (Annville, PA)

$02 / 03 / 86$

Eastman Kodak Co. (Kingsport, TN)

$02 / 17 / 86$

Emory University (Atlanta, GA)

$02 / 18 / 86$

Dupont (Wilmington, DE)

$03 / 11 / 86$

University of Delaware (Newark, DE)

$03 / 12 / 86$

Hoffmann-LaRoche (Nutley, NJ)

$05 / 23 / 86$

ACS Central Regional Meeting Synthesis Symposium (Bowling Green, OH)

$06 / 03 / 86$

BOC Group (Murray Hill, NJ)

$06 / 10 / 86$

University of Paris-Orsay (Paris, France)

$09 / 26 / 86$

XIIth European Colloquium on Heterocyclic Chemistry (Reims, France)

$10 / 01 / 86$

Vanderbilt University (Nashville, TN)

$11 / 11 / 86$

University of Cincinnati (Cincinnati, $\mathrm{OH}$ )

$12 / 05 / 86$

Dickinson College (Carlisle, PA)

$12 / 12 / 86$

Schering Corporation (Bloomfield, NJ)

$02 / 03 / 87$

Oregon State University (Corvallis, OR)

$04 / 20 / 87$

Gordon Research Conference on Heterocyclic Chemistry (New Hampton, NH)

$07 / 06 / 87$

Indiana University of Pennsylvania (Indiana, PA)

$09 / 11 / 87$

Virginia Tech (Blacksburg, VA)

$09 / 24 / 87$

University of Ottawa (Ottawa, Ont., Canada)

$10 / 26 / 87$

University of Maryland (College Park, MD)

$11 / 10 / 87$

Rensselaer Polytechnic Institute (Troy, NY)

$02 / 04 / 88$

SUNY-Albany (Albany, NY)

$02 / 05 / 88$

Wilkes College (Wilkes-Barre, PA)

$02 / 25 / 88$

Abbott Laboratories (Chicago, IL)

$04 / 05 / 88$

Kent State University (Kent, $\mathrm{OH}$ )

$05 / 05 / 88$

Smith-Kline (King of Prussia, PA)

$05 / 10 / 88$

16th IUPAC Conference on Natural Products (Kyoto, Japan)

$06 / 03 / 88$

Sapporo Post-Symposium (Sapporo, Japan)

$06 / 07 / 88$

University of Houston (Houston, TX)

02/07/89

Rice University (Houston, TX)

02/08/89

Texas A\&M University (College Station, TX)

02/09/89

University of Texas (Austin, TX)

$02 / 10 / 89$ 
SUNY-Binghamton (Binghamton, NY)

05/05/89

Fisons Pharmaceuticals (Rochester, NY)

05/26/89

Colgate University (Hamilton, NY)

$09 / 28 / 89$

Hamilton College (Clinton, NY)

09/29/89

University of Virginia (Charlottesville, VA)

$10 / 13 / 89$

SUNY-Stony Brook (Stony Brook, NY)

11/16/89

Rhone-Poulenc Symposium (Research Triangle, NC)

$01 / 22 / 90$

Burroughs-Wellcome (Research Triangle, NC)

$01 / 23 / 90$

Sterling Drug (Rensselaer, NY)

02/08/90

Sterling Drug (Great Valley, PA)

$03 / 26 / 90$

Yale University (New Haven, CT)

03/28/90

Bristol-Myers Squibb Co. (Wallingford, CT)

03/29/90

Royal Society of Chemistry Congress (Belfast, N. Ireland)

$04 / 10 / 90$

Smith-Kline Beecham (Epsom, England)

$04 / 19 / 90$

North Jersey Section-ACS (Roche Institute, Nutley, NJ)

$05 / 21 / 90$

Heterocycles in Synthesis Symposium, ACS Meeting (Washington, DC)

$08 / 27 / 90$

SUNY-Buffalo (Buffalo, NY)

09/19/90

North Dakota State University (Fargo, ND)

$10 / 25 / 90$

University of Minnesota (Minneapolis, MN)

$10 / 26 / 90$

Michigan State University (East Lansing, MI)

$04 / 11 / 91$

Ciba-Geigy Corp. (Ardsley, NY)

$04 / 24 / 91$

American Cyanamid Lederle Labs (Pearl River, NY)

$04 / 25 / 91$

Bristol-Myers Squibb Co. (New Brunswick, NJ)

05/07/91

13th International Congress of Heterocyclic Chemistry (Corvallis, OR)

$08 / 15 / 91$

Pfizer (Groton, CT)

$11 / 07 / 91$

The Pennsylvania State University (University Park, PA)

$01 / 14 / 92$

University of Akron (Akron, $\mathrm{OH}$ )

$03 / 18 / 92$

University of Rochester (Rochester, NY)

04/01/92

Weyeth-Ayerst (Princeton, NJ)

$04 / 29 / 92$

4th Belgian Organic Synthesis Symposium (Leuven, Belgium)

$05 / 26 / 92$

7th Italian Conference on Pericyclic Reactions (Assisi, Italy)

$06 / 02 / 92$

Gordon Research Conference on Heterocyclic Chemistry (New Hampton, NH)

07/06/92

Anaquest (Murray Hill, NJ)

$09 / 15 / 92$

Eastman Kodak (Rochester, NY)

09/30/92 
Smith-Kline Beecham (King of Prussia, PA)

$10 / 29 / 92$

University of Florida (Gainesville, FL)

$11 / 05 / 92$

The Upjohn Company (Kalamazoo, MI)

$03 / 03 / 93$

Parke-Davis (Ann Arbor, MI)

$03 / 04 / 93$

DuPont-Merck Pharmaceutical Company (Deepwater, NJ)

$03 / 31 / 93$

New York Academy of Sciences (New York, NY)

$10 / 05 / 93$

4th French American Chemical Society Meeting (New Orleans, LA)

$01 / 31 / 94$

Brandeis University (Waltham, MA)

$04 / 11 / 94$

University of Missouri (Columbia, MO)

$04 / 22 / 94$

Organic Chemistry Day Symposium, University of Missouri (Columbia, MO)

$04 / 23 / 94$

Utah State University (Logan, UT)

$05 / 04 / 94$

University of Utah (Salt Lake City, UT)

$05 / 05 / 94$

ACS-MARM Symposium, University of Maryland Baltimore County

(Baltimore, MD)

$05 / 25 / 94$

Columbia University (New York, NY)

$11 / 10 / 94$

Scripps Research Institute (La Jolla, CA)

$03 / 24 / 95$

115th Annual Meeting of the Pharmaceutical Society of Japan (Sendai, Japan)

$03 / 29 / 95$

Merck (Rahway, NJ)

$04 / 27 / 95$

Hoffmann LaRoche (Nutley, NJ)

$05 / 11 / 95$

15th International Congress of Heterocyclic Chemistry (Taipei, Taiwan)

$08 / 11 / 95$

Hong Kong International Symposium on Heterocyclic Chemistry (Hong Kong)

$08 / 15 / 95$

Western ACS Meeting, Synthesis Symposium (San Diego, CA)

$10 / 18 / 95$

Northeast Regional ACS Meeting, Synthesis Symposium (Rochester, NY)

$10 / 23 / 95$

Marshall Gates Symposium, University of Rochester (Rochester, NY)

$10 / 28 / 95$

Castle Lecturer, University of South Florida (Tampa, FL)

$01 / 18 / 96$

R.W. Johnson Pharmaceutical Research Institute (Raritan, NJ)

$01 / 25 / 96$

Bayer Pharmaceuticals (West Haven, CT)

$03 / 12 / 96$

Boehringer-Ingelheim Pharmaceuticals (Ridgefield, CT)

$03 / 13 / 96$

Syntex Distinguished Lecturer, Colorado State University (Fort Collins, CO)

$04 / 08 / 96$

University of Illinois (Champaigne-Urbana, IL)

$10 / 09 / 96$

7th Quebec-Ontario Mini Symposium (Waterloo, Ontario, Canada)

$10 / 26 / 96$

Albany Molecular Research (Albany, NY)

Wyeth-Ayerst (Pearl River, NY) 
Biomega-Boehringer Ingelheim (Montreal, Quebec, Canada)

$05 / 29 / 97$

Gordon Conference on Heterocyclic Compounds (Newport, RI)

$06 / 30 / 97$

Agouron Pharmaceuticals (San Diego, CA)

$04 / 07 / 98$

Ligand Pharmaceuticals (San Diego, CA)

04/08/98

New York University (New York, NY)

$04 / 17 / 98$

Rhone-Poulenc Ag Company (Research Triangle Park, NC)

$05 / 26 / 98$

Pfizer (Groton, CT)

$09 / 18 / 98$

8th Symposium on the Latest Trends in Organic Synthesis (Gainesville, FL)

$10 / 31 / 98$

12th Brisbane Organic Chemistry Symposium (Brisbane, Australia)

$11 / 27 / 98$

University of Sydney (Sydney, Australia)

$11 / 30 / 98$

19th Annual One Day Symposium, Royal Australian Chemical Institute

(Sydney Australia)

$12 / 01 / 98$

Research School of Chemistry, Australian National University

$12 / 03 / 98$

(Canberra, Australia)

23rd Annual Synthesis Symposium, Royal Australian Chemical Institute

(Melbourne, Australia)

$12 / 04 / 98$

Adelaide Organic Chemistry Symposium (Adelaide, Australia)

$12 / 07 / 98$

Univeristy of California-Irvine (Irvine, CA)

$02 / 27 / 99$

University of California-Riverside (Riverside, CA)

$02 / 18 / 99$

West Virginia University (Morgantown, WV)

$04 / 24 / 99$

Rhone Polenc Rohrer Visions in Chemistry Symposium

(Collegeville, PA)

$04 / 27 / 99$

Pfizer Central Research (Sandwich, England)

05/04/99

$2^{\text {nd }}$ Sunderland Conference (Sunderland, England)

05/05/99

$14^{\text {th }}$ Lakeland Symposium on Heterocyclic Chemistry

(Grasmere, England)

$05 / 10 / 99$

University of Strathclyde (Glasgow, Scotland)

$05 / 11 / 99$

Procter and Gamble $5^{\text {th }}$ Annual Organic Chemistry Colloquium

(Cincinnati, $\mathrm{OH}$ )

07/30/99

ViroPharma (Exton, PA)

09/09/99

R.W. Johnson Pharmaceutical Research Institute, $11^{\text {th }}$ Annual

Chemistry Lecturer (Raritan, NJ)

$11 / 02 / 99$

Ohio State University (Columbus, $\mathrm{OH}$ )

$01 / 27 / 00$

Florida State University (Tallahassee, FL)

$04 / 04 / 00$ 
Arthur G. Schultz Organic Synthesis Symposium, Rensselaer Polytechnic

Institute (Troy, NY)

$04 / 29 / 00$

Eli Lilly (Indianapolis, IN)

$05 / 16 / 00$

University of Colorado-Roche Synthetic Chemistry Symposium (Boulder, CO)

$05 / 25-26 / 00$

University of Alcala (Alcala de Hennares, Spain)

$07 / 18 / 00$

Gordon Research Conference on Natural Products (Plymouth, NH)

07/30-8/03/00

University of Kentucky (Lexington, KY)

$09 / 01 / 00$

Esther \& Bingham J. Humphrey Memorial Symposium, University of

Vermont (Burlington, VT)

$09 / 30 / 00$

Abbott Laboratories Distinguished Lecture Series (Abbott Park, IL)

$10 / 13 / 00$

The Pennsylvania State University (University Park, PA)

$10 / 26 / 00$

ACS Pacifichem 2000 Heterocycles Symposium (Honolulu, HI)

$12 / 14-19 / 00$

Univeristy of California-Irvine (Irvine, CA)

$01 / 24 / 01$

Univeristy of California-Santa Cruz (Santa Cruz, CA)

$02 / 26 / 01$

Scripps Research Institute (La Jolla, CA)

$03 / 14 / 01$

Allergan Pharmaceuticals (Irvine, CA)

$03 / 16 / 01$

R.B. Miller Lecturer, University of California-Davis (Davis, CA)

$04 / 20 / 01$

Memorial Sloan-Kettering Cancer Center (New York, NY)

$04 / 24 / 01$

GlaxoSmithKline (King of Prussia, PA)

$05 / 03 / 01$

Schering-Plough (Kenilworth, NJ)

$05 / 17 / 01$

R.W. Franck Symposium, Hunter College-CUNY (New York, NY)

$05 / 18 / 01$

Pharmacia Corporation (Kalamazoo, MI)

$06 / 13 / 01$

Gordon Research Conference on Organic Reactions and Processes (Bristol, RI)

$07 / 22-27 / 01$

Sendai Post-ICHC Symposium (Sendai, Japan)

$08 / 04 / 01$

Southeast Regional ACS Meeting Synthesis Symposium (Savannah, GA)

$09 / 24 / 01$

Seoul National University, School of Pharmacy (Seoul, Korea)

$10 / 16 / 01$

$50^{\text {th }}$ Aniversary Meeting Plenary Lecturer, Pharmaceutical Society

of Korea (Seoul, Korea)

$10 / 18 / 01$

Emory University (Atlanta, GA)

$04 / 10 / 02$

North Jersey ACS Spring Symposium on Organic/Medicinal Chemistry

(Woodbridge, NJ)

Bristol-Myers Squibb Co. (Wallingford, CT)

Viropharma, Inc. (Exton, PA) 
Philadelphia Organic Chemists Club Award Symposium (Philadelphia, PA) 10/24/02 AstraZeneca Lecturer

University of Sherbrooke (Sherbrooke, Quebec, Canada)

03/03/03

AstraZeneca (Montreal, Quebec, Canada)

03/04/03

Merck Sponsored Lecturer

University of Leeds (Leeds, UK)

03/17/03

University of Southampton (Southampton, UK)

03/19/03

Merck, Sharp and Dohme (Terlings Park, UK)

03/20/03

GlaxoSmithKline (Stevenage, UK)

03/21/03

University of Texas-Arlington (Arlington, TX)

$04 / 10 / 03$

University of Maryland (College Park, MD)

04/17/03

DuPont Crop Protection Special Organic Seminar (Newark, DE)

06/03/03

P.B.D. de la Mare Lecturer, University of Auckland (Auckland, New Zealand) 02/16/04

University of Canterbury (Christchurch, New Zealand)

$02 / 19 / 04$

26th Gulf Coast Chemistry Conference (Pensacola Beach, FL)

09/09-11/04

University of Arkansas (Fayetteville, AR)

$11 / 01 / 04$

GlaxoSmithKline (King of Prussia, PA)

03/30/05

Indiana University (Bloomington, IN)

04/11/05

GlaxoSmithKline (Research Triangle Park, NC)

$05 / 13 / 05$

ACS Cope Scholar Award Symposium (Washington, DC)

08/30/05

ACS-MARM "Targeted Organic Synthesis" Symposium (Hershey, PA)

06/05/06

Gordon Research Conference on Organic Reactions and Processes

(Smithfield, RI)

07/16-21/06

Gordon Research Conference on Natural Products (Tilton, NH)

07/23-28/06

Schering-Plough (Union, NJ)

08/22/06

$9^{\text {th }}$ Florida Heterocyclic Chemistry Conference (Gainesville, FL)

03/10-13/08 